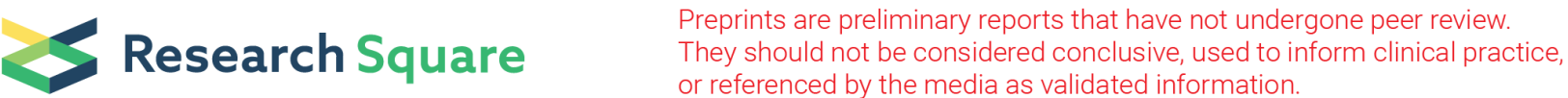

\section{A Qualitative Study of the Development and Utilization of Health Facility-based Immunization Microplans in Uganda.}

\section{David Kaawa-Mafigiri ( $\sim$ mafigiridk@yahoo.com )}

Makerere University College of Humanities and Social Sciences https://orcid.org/0000-0002-26994248

\section{Constance Iradukunda}

Center for Social Sciences Research on AIDS, Makerere University

\section{Catherine Atumanya}

Center for Social Sciences Research on AIDS, Makerere University

\section{Michael Odie}

Center for Social Sciences Research on AIDS, Makerere University

\section{Arielle Mancuso}

World Health Organization

Nhan Tran

World Health Organization

Janet McGrath

Case Western Reserve University

Henry Luzze

Republic of Uganda Ministry of Health

\section{BMC Supplements Reviewed}

Keywords: Microplan, Utilization, Routine Immunization, Qualitative Study, Uganda

Posted Date: March 26th, 2021

DOl: https://doi.org/10.21203/rs.3.rs-326895/v1

License: (c) (1) This work is licensed under a Creative Commons Attribution 4.0 International License. Read Full License

Version of Record: A version of this preprint was published at Health Research Policy and Systems on August 11th, 2021. See the published version at https://doi.org/10.1186/s12961-021-00708-y. 


\section{Abstract}

Background: In 2006, Uganda adopted the Reaching Every District strategy with the goal of attaining at least $80 \%$ coverage for routine immunizations in every district. The development and utilization of health facility/district immunization microplans is the key to the strategy. A number of reports have shown suboptimal development and use of microplans in Uganda. This study explores factors associated with suboptimal development and use of microplans in two districts in Uganda to pinpoint challenges encountered during the microplanning process.

Methods: A qualitative study was conducted comparing two districts, Kapchorwa with low immunization coverage and Luwero with high immunization coverage. Data were collected through multilevel observation of health facilities, planning sessions and planning meetings; records review of microplans, micromaps, and meeting minutes; 57 interviews with health workers at the Ministry level and lower cadre health facility workers. Data were analyzed using NVivo 8 qualitative text analysis software. Transcripts were coded, memos and display matrices were developed to examine the process of developing and utilization of microplans, including experiences of health workers (implementers).

Results: Three key findings emerged from this study. First, there are significant knowledge gaps about the microplanning process among health workers at all levels (community and district health facility and nationally). Limited knowledge about communities and program catchment areas greatly hinders the planning process by limiting the ability to identify hard-to-reach areas as well as prioritize areas according to need. Secondly, the microplanning tool is bulky and complex. Finally, microplanning is being implemented in the context of already over-tasked health personnel who have to conduct several other activities as part of their daily routines.

Conclusions: In order to achieve quality improvement of the Reaching Every District campaign, the microplanning process should be revised. Health workers' misunderstanding and limited knowledge about the microplanning process, especially at peripheral health facilities, coupled with the complex, bulky nature of the microplanning tool reduces the effectiveness of microplanning in improving routine immunization in Uganda. The study reveals the need to reduce the complexity of the tool and identify ways to train and support workers in the use of the revised tool, including support in incorporating the microplanning process into their busy schedules.

\section{Background}

Routine immunization (RI) is the backbone of immunization as a public health tool [1] - a fundamental service intended to reach all children with multiple doses of vaccines. In 2002, the World Health Organization (WHO) developed the Reaching Every District (RED) strategy which aimed to improve vaccination coverage globally. Following introduction of the RED approach, RI coverage improved in many low-income countries. However, coverage has stalled since 2008-2009 and globally by 2017 an estimated 19.9 million infants were not reached by routine immunization services [2] . 
In 2006, Uganda adopted the RED strategy with the goal of attaining at least $80 \%$ coverage for routine immunizations in every district across the country. In Uganda, the RED strategy evolved, as it has in many countries, to become Reaching Every Community/Child (REC)[1,3]. The approach proposes five operational strategies; 1) Re-establishing health outreach where it has been dismantled. Outreach is any strategy that requires health facility staff to leave their facility to deliver immunization. Ideally a minimum of five contacts per year is required to fully immunize an infant in Uganda; 2) Supportive supervision, defined as provision of regular onsite training to health workers. The supervision helps to solve problems locally and follow up supply and resource issues; 3 ) Linking services with communities so as to meet specific local needs. The community should be encouraged to take ownership of planning and service delivery; 4) Monitoring and use of data for action. This involves timely collection of data at facility and district level and use of the data to solve problems; and 5) Planning and management of resources.

To operationalize the REC strategy, a health facility/district microplanning tool was developed to guide health workers in planning and delivering immunization services [1]. An effective microplan aims to reach all target populations with immunization services as recommended in the vaccination schedule. The microplan should be based upon a local situation analysis which involves every health facility and through them the community that they serve. It includes actions for improving coverage and quality of services. REC microplans have well defined catchment areas and realistic local solutions for operational, economic, political and social barriers to immunization [1]. The microplan describes the demography of the health facility catchment area by listing villages and parishes (parish is an organizing administrative structure comprising a number of villages along which service delivery is planned. Several villages make up a parish and alternatively several parishes make up a county and district) in the catchment area, total population per village and the target populations (children under 1 year, children under 5 years, pregnant women) [1]. It includes a sketch of health facility catchment area with all routine immunization points (both static and outreaches) and geographical features e.g., rivers, swamps and mountains. Other topical areas the microplan captures are a mapping of immunization service points, both static and outreaches, showing how villages in the catchment area are served. Additionally, the microplan entails documentation on performance monitoring and, drop-out rate monitoring charts that are updated monthly. The microplan also has section on social mapping indicating stakeholders and partners in villages and parishes (parish is an organizing administrative structure comprising a number of villages along which service delivery is planned). Finally there is a section showing the plans and budget needed for implementation of the microplan.

Despite having adopted the RED strategy in 2006 , by 2015 , only 40 of the 112 districts had updated microplans. No systematic assessment has been conducted to assess underlying factors contributing to sub-optimal development and use of microplans in Uganda. This research examined the process and challenges of developing and utilizing microplans for improving immunization outcomes in Uganda. In addition, this research identified gaps and explored strategies to improve the microplanning process in two districts in Uganda. By comparing a "high performing" and "low performing" district, the study was positioned to better understand the development and implementation of the microplan process unfolded and associated barriers to its adoption. The knowledge obtained from this work suggests strategies to 
address challenges confronting the microplanning process, with the overall aim of improving immunization rates at health facility and district levels.

\section{Methods}

\section{Design and setting}

This qualitative study entailed multiple methods to examine the process of developing and employing microplans in Luwero (with high immunization coverage) and Kapchorwa (low coverage) districts in Uganda[4]. Luwero district is located in Central Uganda, approximately $75 \mathrm{KM}$ (47 miles) by road north of Kampala Capital City. Luwero is covered with savannah and characterised with a tropical kind of environment. Agriculture is the main economic activity with coffee and bananas comprising the major cash crops. Kapchorwa district is located in Eastern Uganda $287 \mathrm{KM}$ east from Kampala Capital City. Kapchorwa is generally hilly with steep slopes and valleys with several rivers flowing from Mt. Elgon. As such Kapchorwa is covered with mixed mountainous forests and less open savannah compared to Luwero. Kapchorwa is sparsely populated due to both the terrain that makes it more hazard prone to rock and landslides and a history of insecurity arising from cattle rustling practices. The main economic activity is agriculture with coffee, millet and potatoes comprising the majority of crops farmed and traded. Majority of the population in both districts is made up of children.

Whereas several districts at the time of the study had rolled out with the RED programme, both Luwero and Kapchorwa districts were among the first districts to have participated in the pilot program when the intervention was being tested.

As such they both had undergone much more exposure to the use of microplans. Both districts were selected purposively given their unique setting and characteristics to represent the variability of population characteristics in Uganda. Methods included multilevel observation of health facilities, planning sessions and planning meetings; records reviews of microplans, micromaps, and meeting minutes; and interviews. The 2014 WHO/UNICEF joint report states that the national immunization coverage in Uganda, as measured by the third dose of Diphtheria, Pertussis, Tetanus, Hepatitis B and Haemophillus influenza vaccine (DPT3) was $100 \%$ [5]. Luwero district with a total population of 467,466 and 20,101 children under 1 year and 65 health facilities reported DPT3 coverage of $115 \%$. Kapchorwa district, with a population of 121,163 and 5,210 children under 1 year and 20 health facilities had DPT3 coverage of only $66 \%$ [5]. Luwero is more populated and has many more health facilities compared to Kapchorwa, the study purposively selected nearly all facilities in Kapchorwa to increase the representation of experiences given the vast expanse and variability within Kapchorwa district.

Health facilities in Uganda are classified into seven levels based on the services they provide and the catchment area they serve. The health facilities are designated as Health Centre Level one (HC I) to Health Centre Level four (HC IV); General Hospital, Regional Referral Hospital and National Referral Hospital[6]. In Uganda, immunization is part of the integrated minimum health care package delivered by local governments at the district level. Therefore, responsibility for the delivery of health services, 
including immunization, rests with local district governments, each operating under the mandates of the national health system. At the district level, the health system is composed of different structures where the lowest level within the community is Health Centre I ( $\mathrm{HC} 1$ ) (composed of Village Health Team members) followed by Health Centres II, III, IV and General Hospital respectively. This ranking is based on population size in the catchment area, ranging from HC1s where each VHT serves approximately 1000 persons or 25 households in less densely populated areas. This ranking corresponds to the population size reflected by the local administrative structures and covers similarly larger populations as one moves along the different levels of hospitals and administrative structures. Presently, under the RED strategy, all health facilities from Health Centre II and above in the district are expected to have community - based microplans for immunization. Study participants were selected to represent the four levels comprising a district catchment area. Notably, some participants at the district level also represented the National level as they serve in policy and planning at the National Ministry level by virtual of their offices held at District level. Fifty-two public health facilities in both districts ( $n=17$ in Kapchorwa) were examined in this study for microplan utilization patterns using in-depth interviews as well as review of documents (Facility records e.g. maps, meeting minutes, and immunization record books). Notably, 9 of the health facilities examined ( $n=4$ in Kapchorwa) were private NGO facilities that engage in immunization activities implemented by the Ministry of Health. Health facilities were also purposively selected for the study aiming to cover both rural and urban settings in both districts. In both districts, the study focused on different stakeholders at the selected sites namely: (1) health workers at both public and private health facilities involved in immunization activities including National level EPI managers, District level EPI managers, health facility in-charges, health workers at the facility level; (2) community members particularly those involved in child care who may be responsible for seeking immunization services in the two regions including district level administrators Chief Administrative Officers, District Local Council Chairpersons, and area Members of Parliament who influence immunization programs.

\section{Data collection}

The study relied on purposive sampling to determine the participants to interview. Social science research assistants (RAs) with prior experience in qualitative data collection techniques conducted 57 key informant interviews ( $n=17$ in Kapchorwa and $n=40$ in Luwero) with health workers responsible for immunization services. Official letters were written by the Principal Investigators informing the districts of the project and introducing the study team. The district health personnel were also provided with copies of the Makerere University's ethical clearance letters that were attached to the PI's introductory letter. A designated member of the district health team (DHT) in each district worked with the project team to identify participants from the health facilities and the community within the catchment area of a particular health facility. Key informants were approached by an RA and invited to participate. Enrolment of participants and data collection continued until saturation was reached. Interviews focused on several domains: experiences, knowledge of and practices during the process of developing the microplans, successes and challenges encountered during the microplanning process. Two study investigators (DKM and $\mathrm{HL}$ ) reviewed health facility records including immunization monitoring charts, catchment area maps, outreach and District Health Office support supervision notes. 
Review of this data generated comparative data on level of knowledge and key landmarks such as critical events (social mobilization activities), individuals, or documents utilized during implementation of microplans. During data collection, all study investigators (DKM, HL and JWM) monitored all data collection processes to ensure that the process complies with ethical and quality requirements of the study.

\section{Data Management and analysis}

All interviews and field notes were transcribed verbatim and translated to English by the RAs. Thereafter the data were analyzed using NVivo QSR qualitative text analysis software [7]. Transcripts were read through so that the researchers were familiar with the data. Then related categories were sourced out. For each category, we identified a number of codes where each served as an indexing or measurement device to assign values to the text and help organize the data. These data were first coded by two RAs and reviewed by one study investigator (DKM) for uniformity before all coding was complete. A codebook was developed and pilot tested on five KII transcripts. Thereafter topical and thematic codes were applied to all transcripts and field notes following a content-driven theme approach [8-10]. Codes were synthesized into categories and themes, while variables were compared across levels of analysis. Findings from interview data were corroborated with summaries generated from review of various relevant documents and notes from observations made at health facilities. This process was to ensure accuracy and increase validity of emerging themes.

\section{Results}

Fifty-seven participants were drawn from different health facilities in both districts ranging from Health Centre (HC) I/IIs, HC III, HC IV and hospitals. Table 1 below illustrates the type of respondents by their job title, facility level they were found and district. 
Table 1

Type of health workers interviewed by facility and district $(\mathrm{N}=57)$

\begin{tabular}{|lll|}
\hline Job Title & Luwero $(\mathbf{N}=40)$ & Kapchorwa $(\mathbf{N}=17)$ \\
\hline Administrative Staff & 1 & 0 \\
\hline Medical doctor and Clinical Officer & 3 & 1 \\
\hline Nurse and Midwife & 25 & 13 \\
\hline Nurse aide and Health Assistant & 7 & 2 \\
\hline Others (e.g. Lab tech, Drug dispenser) & 4 & 1 \\
\hline Type of health facility & & \\
\hline Health Centre I/II & 13 & 4 \\
\hline Health Centre III & 20 & 13 \\
\hline Health Centre IV & 5 & 0 \\
\hline Hospital & 2 & 0 \\
\hline
\end{tabular}

At the time of the study, the average time a health worker had served at their current post was 4.5 years (range one month -36 years). Over $90 \%$ of the health workers reported that prior to engaging in the microplan development and utilization process they worked at a different health facility, were at school or were volunteers at the facility. As such, the microplanning process in specific catchment areas was being implemented by relatively 'new' health workers to that area. One third (36\%) of health workers at the district level facilities had not been involved in the microplanning process prior to their current job. Most facilities (95\%) reported having a health information system through which they monitored their immunization activities. Slightly more than half $(57 \%)$ of the facilities had an updated microplan in place. Notably, very few (27\%) privately owned health facilities that participate in routine immunization activities had updated microplan in place compared to public $(71 \%)$ and NGO/FBO $(60 \%)$ owned facilities. According to the records reviewed about the National Immuniazation Programme, in Luwero district, the proportion of facilities with updated microplans was reported as $65 \%$ compared to $57 \%$ in Kapchorwa district.

\section{Health Workers' understanding about microplans and microplanning process}

Majority of health workers $(\mathrm{N}=40)$ were generally aware of the presence and importance of microplans and the microplanning process in their health facilities. They further acknowledged the significant roles of microplans in identifying and solving several health challenges within communities, at health facility, district and national levels. Overall, while most health workers were aware of the presence and intended benefits of microplans as a means to improve health service delivery, many of them in Health Centres III 
and II $(n=15)$ had never participated in the microplannning process and did not understand how to implement the microplans.

"Microplanning? I don't know its meaning. First tell me the meaning of microplanning, then I will answer." Nurse at Luwero, Kasozi HC III

"I have not much information but what I understand deals with taking drugs which are adequate to the health facilities." Chemosong Health Centre II, Kapchorwa

\section{Catchment area}

Notably, some health workers $(\mathrm{N}=12)$ at several health facilities admitted not comprehending some issues concerning their catchment area. For instance, being unsure of their catchment population emerged as a major hindrance in the development of the microplan.

\section{Training of health workers}

While most health workers mentioned that they were not trained and did not have specific roles in the microplanning process, other health workers within these health facilities received training on microplanning. Those who received training mostly included facility in-charges, EPI focal persons, adolescent health focal persons and midwives. As a result, these health workers were often the ones considered to have specific roles and responsibilities in the microplanning process. Participants also added that microplanning trainings were carried out at different phases and they were not certain of the training content. Notably, data reveal that some of the training and capacity building activities were not well coordinated.

For example, twenty three participants indicated the process was characterized by various formats including workshops, one-on-one sessions or briefs provided by colleagues who had returned from a training session.

Some participants perceived the time allocated to learn about the microplanning process to be insufficient, as stated by this participant:

I went to a HC (health centre), joined people being oriented and picked a few things on MP (microplan). Most of us were mentored on MP for 2 hours only Nurse at Kapchorwa, Kaserem HC III

\section{Information system for construction of microplans}

Majority of health workers $(\mathrm{N}=42)$ identified various sources of information for the microplanning process including outreach activities, family planning activities, youth friendly services, population and demographic data of villages, parishes and sub-counties as well as records of different health activities. Health workers further cited specific information necessary to construct microplans such as identifying areas to be prioritized and planned for, ascertaining favourable days for outreaches, classifying facilities to be covered and following the work plan. 
"We had first the general map of the service area. We got the target population per month. We also used the distance of the outreach post to the facility. We also allocated the number of staff to go for the outreach and facility. We also allocated the number of staff to go for the outreach, and facilitation for each staff. We also noted the specific mobilizers per post." Kapchorwa, Kaserem HC III

"Every month we look at it to know how many have completed the dose and have defaulted. We refer to it every month to know why some mothers default, why some mothers have not brought their babies." Senior Medical Officer, Luwero HC IV

Despite knowledge about the importance of having information for constructing microplans, health workers revealed that accessing relevant information remains challenging as most health records are not integrated in the health information management system of health facilities and sometimes records are misplaced before they can be archived. Additionally, lack of updated information necessary to construct microplans was cited as a major hindrance to the MP process. As a result, there is limited knowledge about communities and program catchment areas, which greatly affects the planning process. For example health facilities' ability to identify hard-to-reach areas as well as prioritize areas according to need is limited. The microplanning tool was also perceived to be bulky (15 to 17 pages) and complex to complete. This is in context of an already over-tasked health workforce, especially at the lower-level health facilities.

\section{Importance of the microplan}

All health workers mentioned that microplans are essential for effective service delivery although some recognized that microplans are more easily planned and implemented at higher level health facilities like $\mathrm{HC}$ IIIs and IVs because of the presence of better qualified staff, health equipment and generally a more organized health system.

Some participants perceived that microplans have a significant impact on delivery of health care services, particularly child immunization. Most participants $(\mathrm{N}=30)$ mentioned that microplans help ease requisition of vaccines, get information about new vaccines, provide health education to communities about the importance of immunizing children, and estimate vaccine stock outs.

"Microplan gave us the number of villages to be covered at the outpost".

Laboratory Technician at Kapchorwa, Cheptuya HC III

From overall populations we decided, come up with target population per post, per month as well. It helps in ordering the quantity of vaccine according to the number of children Nurse at Kapchorwa, Kaserem HC III

Some participants also mentioned that the microplan guides health workers on what has to be accomplished and timelines for planned activities thus enabling them to identify barriers to immunization activities and identify solutions. Such data were reportedly used to avoid vaccine stock-outs as well as 
plan for subsequent health programs. Additionally, some respondents also noted that microplans ease health workers' duties since they will know when a particular activity is to be implemented, who will implement it, who the target population are and the potential impact of the activity.

"....how you can plan to carry out the activities of immunisation so that it can run smoothly to reach the community and to see whether the immunisation exercise can reach up to the grassroot." Partner NGO Administrator and Member of Health Ministry Technical Working Group on Immunization

\section{Discussion}

Our findings show that only $57 \%$ of the health facilities had updated microplans and further describe health workers' perceptions, knowledge and challenges about the microplanning process. Factors that contribute to lack of updated microplans in some facilities include: health workers' limited knowledge about the MP process, especially at the peripheral health facilities, the complex, bulky nature of the MP tool, and inadequate information on the catchment area and catchment population.

Despite having introduced the microplanning tool over 10 years ago, knowledge gaps were identified among health workers that are supposed to use the tool on a day-to-day basis. The major reasons for the low knowledge include staff attrition, recruitment of new staff and targeting only some specific health workers for training. To bridge the knowledge gap, we propose that future training should include all health workers at the facility and should provide a standard 'How to do' guide.

The 'how-to do' guide should be designed targeting the health facility workers at the lower levels who appeared to face more challenges in adapting their practice and knowledge to optimally utilize the microplan process.

In the long term, microplanning should be integrated in the pre-service curriculum so that all newly qualified health workers acquire the knowledge.

Currently, there are efforts to integrate other programs like maternal health, nutrition, and child health, into the microplanning tool. In Mongolia, the microplanning tool was shown to have wider health system applications especially in responding to maternal and child health service needs [11]. Integration of these programs into the immunization microplanning tool would require involvement of other health workers within the facility, hence reducing the burden of developing the tool by immunization staff. However we note the potential risk of overburdening the very health workers struggling to adapt the microplan due to it being bulky should the integration not be done cautiously. The already overburdened health workers at the lower level health facilities would need to carefully consider how best to integrate other services planning processes with the microplan process.

Knowledge of the health facility catchment area is a critical starting point of a good microplan [1, 11-12]. In this study, health workers expressed inadequate information about catchment area and catchment population as a major hindrance to the development and utilization of the microplans. The process of ascertaining the catchment area for each health facility should be initiated at the district level with 
involvement of the planning department and local authorities to ensure that all areas are adequately served. Use of geographic information system (GIS)-based maps to clearly demarcate catchment areas can improve the quality of the microplan. Notably, improved vaccination coverage was demonstrated in Nigeria through the use of GIS [13-14]. Our data further support the need to tailor the microplanning process to the local context to optimally utilize microplans [15-17]. In some health facilities where the catchment area was known, clients still preferred to obtain services from other facilities outside the designated catchment area depending on convenience. In China, the Integrated Catchment Area approach that takes into account people's actual behaviors improved accessibility to healthcare facilities when compared to the traditional catchment area methods [18]. The bulkiness and complexity of the microplanning tool was cited as another hindrance in its development and utilization. The tool can be reviewed and reduced from the current 17 pages, but also ensuring that content is not lost. The Ministry of Health's immunization programme staff should devise easier forms that capture information that is easy to tally at the lower level health facilities for onward compilation at the district and national level. The tallying process should be intertwined with more frequent support supervision visits by the higher level health workers.

We note that whereas challenges to utilization of the microplan were noted in both districts, Luwero's immunization coverage was higher than that of Kapchorwa. This may point to other factors beyond the implementation of the microplan influencing routine immunization. We caution that any conclusion about the impact that inability to optimally utilize microplanning may have on low coverage take into account the potential effect of social and economic differences between the districts. More research needs to examine plausible causes of differences in the coverage between both districts.

\section{Conclusion}

Our findings point to longstanding challenges to the microplanning process that hinder its development and utilization particularly in rural districts in Uganda. To sustainably train health workers on the microplanning tool, there is need to involve more than one off sessions, and continuous education and support supervision. There is also need to review the microplan tool to make it easier to utilize particularly for lower level, rural based health workers. For instance, in addition to a less bulky and complex tool, the Ministry of Health should introduce 'how to do' training and guides to standardize the process of microplan development and use. Once properly developed, the microplanning tool can be used to reach every child and effectively improve vaccination coverage.

\section{Abbreviations}

DHT - District Health Team

DPT3 - Diphtheria, Pertussis, Tetanus, Hepatitis B and Haemophillus influenza vaccine EPI - Expanded Programme on Immunization 
FBO - Faith Based Organization

FGD - Focus Group Discussion

GIS - Geographic Information System

HC I - Health Centre Level one

HC II - Health Centre Level two

HC III - Health Centre Level three

HC IV - Health Centre Level four

HDREC - Higher Degrees and Research Ethics Committee

MP - Microplan

NGO - Non-Governmental Organization

$\mathrm{PI}$ - Principal Investigator

RAs - Research Assitants

REC - Reaching Every Community/Child

RED - Reaching Every District

RI - Routine Immunization

UNCST - Uganda National Council for Science and Technology

UNICEF - United Nations Children's Fund

WHO - World Health Organization

WHO ERC - World Health Organization Research Ethics Review Committee

\section{Declarations}

\section{Ethics approval and consent to participate}

The study was approved by the World Health Organization Research Ethics Review Committee (WHO ERC.0002685); Makerere University School of Public Health Higher Degrees and Research Ethics Committee (HDREC 360) and registered by the Uganda National Council for Science and Technology (UNCST SS 3969). The study team was certified in human subjects protection. Written informed consent was obtained from each individual participant for all interviews and FGDs. Data were centrally owned 
and managed by the Principal Investigators who were responsible for access to the data during the entire duration of the project.

\section{Consent for publication}

Written, signed informed consent was obtained from participants for all interviews and FGD data to be published.

\section{Availability of data and materials}

The datasets used and/or analysed during the current study are available from the corresponding author on reasonable request.

\section{Competing interests}

The authors declare that they have no competing interests.

\section{Funding}

This study was funded through the Alliance for Health Policy and Systems Research, Health Systems and Innovation of the World Health Organization. The Alliance for Health Policy and Systems Research, Health Systems and Innovation of the World Health Organization facilitated pre and post research workshops that included discussions about the design, and dissemination of findings respectively. They did not have any involvement in the research implementation activities. Publication costs are funded by The Alliance for Health Policy and Systems Research, Health Systems and Innovation of the World Health Organization.

\section{Authors' contributions}

DKM, Cl, CA, MO, AM, NT, JWM, HL designed the study, analyzed and interpreted data regarding microplan implementation processes.

DKM, AM, NT, JWM, HL were major contributors in writing the manuscript.

All authors read and approved the manuscript.

\section{Acknowledgements}

The authors acknowledge the contributions to this project from UNICEF, Alliance for Health Policy and Systems Research, Health Systems and Innovation Team, the Ugandan Ministry of Health's Expanded Programme on Immunization, the research assistants and study team, and the study participants. We also appreciate the anonymous reviewers who provided feedback on this manuscript draft.

\section{References}


1. WHO, UNICEF. Microplanning for immunization service delivery using the Reaching Every District (RED) strategy. Geneva: World Health Organization2009.

2. Immunization coverage fact sheet [database on the Internet]. World Health Organization. 2018 [cited 14 May 2019]. Available from: https://www.who.int/news-room/fact-sheets/detail/immunizationcoverage.

3. Bilous VJ, Nshimirimana J D. Reaching Every District (RED) approach: a way to improve immunization performance. Bulletin of the World Health Organization. 2008 Mar,86(3):A-b.

4. Uganda Bureau of Statistcs (UBOS), ICF. Uganda Demographic and Health Survey 2016: Key Indicators Report. Kampala, Uganda and Rockville, Maryland, USA: Uganda Bureau of Statistics2017.

5. WHO, UNICEF. Uganda: WHO and UNICEF estimates of immunization coverage: 2014 revision. Geneva: World Health Organization2015.

6. Ministry of Health. Health Facilities Inventory, July 2012. Kampala, Uganda: Ministry of Health2013.

7. QSR International. NVIVO 8 for Windows. [cited 20 Feb 2017]. Available from: https:// www.qsrinternational.com/nvivo/contact-us: QSR International Pty Ltd; 2010.

8. Babbie E. The Practice of Social Research. 9 ed. Belmont, CA: Wadsworth/Thomson Learning; 2001.

9. Patton M. Qualitative Evaluation and Research Methods. Newbury Park, CA: Sage Publications; 1990.

10. Bernard HR. Research methods in anthropology: qualitative and quantitative approaches. 3 ed. Walnut Creek: AltaMira Press; 2002.

11. Enkhtuya B, Badamusuren T, Dondog N, Khandsuren L, Elbegtuya N, Jargal G, et al. Reaching every district - development and testing of a health micro-planning strategy for reaching difficult to reach populations in Mongolia. Rural Remote Health. 2009 Apr-Jun;9(2):1045.

12. LaFond A, Kanagat N, Steinglass R, Fields R, Sequeira J, Mookherji S. Drivers of routine immunization coverage improvement in Africa: findings from district-level case studies. Health policy and planning. $2015 \mathrm{Apr}, 30(3): 298-308$.

13. Barau I, Zubairu M, Mwanza MN, Seaman VY. Improving polio vaccination coverage in Nigeria through the use of geographic information system technology. J Infect Dis. 2014 Nov 01;210 Suppl 1:S102-10.

14. Gammino VM, Nuhu A, Chenoweth P, Manneh F, Young RR, Sugerman DE, et al. Using geographic information systems to track polio vaccination team performance: pilot project report: Improving polio vaccination coverage in Nigeria through the use of geographic information system technology. J Infect Dis. 2014 Nov 1;210 Suppl 1:S98-101.

15. Babirye JN, Engebretsen IM, Rutebemberwa E, Kiguli J, Nuwaha F. Urban settings do not ensure access to services: findings from the immunisation programme in Kampala Uganda. BMC health services research. 2014 Mar 06;14:111.

16. Haddad S, Bicaba A, Feletto M, Taminy E, Kabore M, Ouedraogo B, et al. System-level determinants of immunization coverage disparities among health districts in Burkina Faso: a multiple case study. BMC international health and human rights. 2009 Oct 14;9 Suppl 1:S15. 
17. Levin A, Kaddar M. Role of the private sector in the provision of immunization services in low- and middle-income countries. Health policy and planning. 2011 Jul;26 Suppl 1:i4-12.

18. Pan X, Kwan MP, Yang L, Zhou S, Zuo Z, Wan B. Evaluating the Accessibility of Healthcare Facilities Using an Integrated Catchment Area Approach. Int J Environ Res Public Health. 2018 Sep 19;15(9). 